\title{
Las yeserías del Patio del Sol del Real Alcázar de Sevilla: Caracterización y cronología
}

\author{
The plasterwork of the Courtyard of the Sun of the Real \\ Alcázar de Sevilla: Characterization test and chronology
}

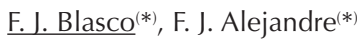

RESUMEN

En esta investigación se realiza un estudio interdisciplinario que tiene como finalidad discernir la originalidad de las yeserías mudéjares del Patio del Sol del Real Alcázar de Sevilla. Para ello se ha trabajado con una doble metodología: una histórica sintética, basada en el análisis y relación de la documentación gráfica e historiográfica existente de este Patio, y otra de caracterización del material de las yeserías, mediante las técnicas instrumentales de FRX, DRX, datación por radiocarbono de fibras vegetales y el análisis gravimétrico del residuo insoluble en agua. La comparación de la composición química, mineralógica y porcentaje de residuo insoluble realizado a tres muestras de yeserías en las que se han observado distintas texturas, sumado a la datación mediante C-14 efectuada a una de ellas, junto al conocimiento de las fuentes históricas y documentales, han permitido identificar y acotar las zonas originales mudéjares de los siglos XIII y XIV y las restauradas del siglo XX.

\section{SUMMARY}

This research involves an interdisciplinary study aimed at determining the origin of the Mudéjar plasterwork of the Patio of the Sun in the Royal Alcázar of Seville. A double methodology has been used involving, on one hand, a synthetic history based on the analysis and correlation of the graphic and historiographic documentation of this Patio and, on the other hand, a characterization of the plasterwork material using $X R F, X R D$, radiocarbon dating of vegetable fibres, and the gravimetric analysis of the water-insoluble residue. Combining the results from the chemical and mineralogical compositions and the percentage of insoluble residue performed on three plasterwork samples with different textures, from C-14 dating of one sample, and from historical and document sources has allowed us to identify and delimit the original Mudéjar zones from the 13th and 14 th centuries from the areas restored in the 20th century.
Palabras clave: Real Alcazar; yeserías; patrimonio; caracterización; conservación.
Keywords: Real Alcazar; plasterworks; heritage; characterization; conservation.

\footnotetext{
(*) Universidad de Sevilla (España). 
1. Arquería mudéjar del antiguo cuarto del Alcaide y columna de la arquería del siglo XVIII.

\section{INTRODUCCIÓN}

En el Patio del Sol del Real Alcázar de SeviIla se halla una arquería mudéjar (s.XIIIs.XIV) que fue descubierta y restaurada por R. Manzano (Director Conservador del Alcázar entre 1970 y 1991). Dicha arquería está decorada con unas yeserías del mismo estilo, de las que no se ha hallado documentación relativa a esta intervención y que visualmente presentaba paños con leves diferencias texturales y de color, que apuntaban a que no todos fueran originales mudéjares. Sobre esta hipótesis se ha realizado un estudio interdisciplinario con el objetivo de aclarar las incertidumbres de adscripción surgidas como consecuencia de esta intervención sin documentar, trabajando con una metodología histórica sintética, y otra de caracterización del material componente de las yeserías.

Analizando las fuentes históricas del Real Alcázar de Sevilla y de las intervenciones realizadas en él, puede hallarse bastante información referente a su construcción y reformas (1) (2), aunque se ha encontrado muy poca con respecto a las yeserías mudéjares del Patio del Sol (1) (3). Ante esta dificultad se ha seguido un método histórico sintético, relacionando los escasos datos existentes y aparentemente aislados, tratando de unificar las diversas informaciones. Simultáneamente se han tomado muestras representativas de las distintas texturas observadas visualmente en las yeserías del patio, caracterizándolas por separado para que en base a los resultados obtenidos, se puedan establecer relaciones y diferencias entre ellas, que permitan acotar las áreas originales y las restauradas de las mismas.

El origen del Alcázar, lo podemos situar en la época del primer Califa andaluz Abd al-Rahman III, hacia el 913. Al Mutamid amplió el recinto y construyó el palacio "Al Mubarak" durante el siglo XI, levantándose posteriormente con los Almohades (siglos XII-XIII) el palacio conocido como del Yeso. Tras la reconquista, Alfonso X edificó el Palacio Gótico en 1254, Alfonso XI la Sala de la Justicia y Pedro I el palacio Mudéjar entre 1364 y 1366. Todo el palacio se enriquecería en los s.XV y XVI durante los Reyes Católicos, seguido de períodos de esplendor y de decadencia durante el s.XVII, al que le siguieron momentos de lujo durante los borbones y de desastres tras el terremoto de Lisboa de 1755. Tras diversos desatinos durante el reinado de Fernando VII en el s.XVIII y algunas intervenciones durante el reinado de Isabel II durante el s.XIX, Ilegamos a las obras realizadas en el monumento por el arquitecto restaurador Rafael Manzano, durante las décadas de los 60 y 70 del siglo XX (1) (3), y a las distintas actuaciones de conservación, restauración o remodelación llevadas a cabo por distintos investigadores (4).

Sabemos que el Patio del Sol ocupa el frente norte del patio de la Alcobilla y debe su fisonomía actual a una profunda remodelación desarrollada en la década de los 70 del s.XX, siendo siempre un espacio doméstico muy abandonado de la propia vivienda del alcaide (1), que ocupaba la parte alta del apeadero (hoy salón de exposiciones). Este ala izquierda del apeadero, que daba al patio del tenis, al patio del Alcaide y al patio del Sol antiguo, lo convirtió en vivienda R. Manzano, organizando aquellos patios y llevando a cabo una exploración arqueológica que permitió encontrar los restos mudéjares del antiguo cuarto del Alcaide que son incluso más antiguos que los del palacio de don Pedro y una arquería del siglo XVIII (3) (Figura 1).

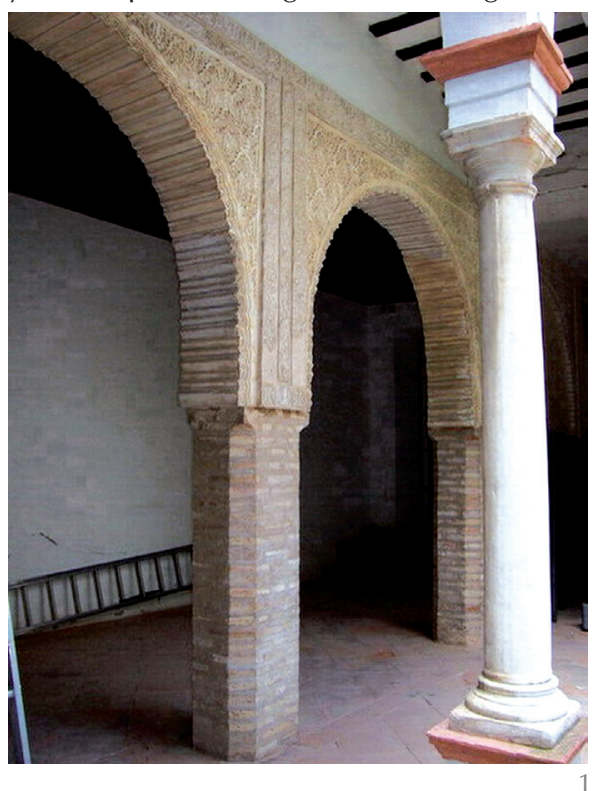

Basilio Pavón Maldonado (5), nos describe una enjuta con motivo agallonado y flor de loto perteneciente a este patio como yesería mudéjar del siglo XIII del arco del apeadero, identificada y fotografiada por los autores de este trabajo (Figura 2).

El mudéjar en su origen y desarrollo es adopción cristiana del arte árabe andaluz al que se le fueron añadiendo improntas del pasado árabe sevillano y nuevas aportaciones cristianas, es decir, un arte ecléctico en permanente evolución, síntesis del arte árabe y cristiano. En el mudéjar sevillano se evidencia un almohadismo tardío, con reflejos abbadíes y califales.

Del Alcázar existen estudios relacionados de alguna forma con las yeserías del monumento: 


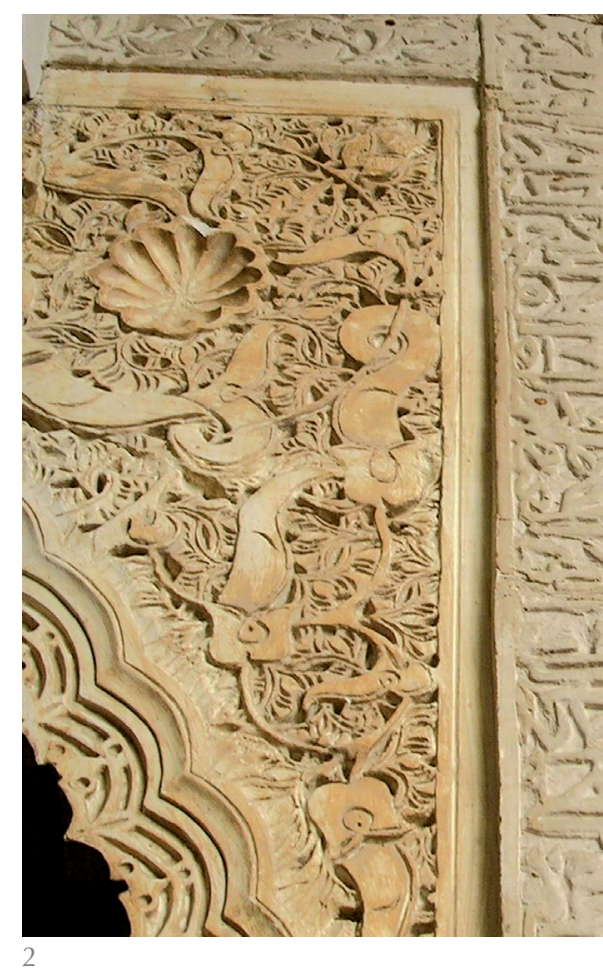

- En función de la evolución histórica de su construcción (1) (2).

- De sus pinturas murales como soportes de las mismas (6).

- De tipo arqueológico, donde a través de excavaciones y estudios murarios se han obtenido muestras de este material (7).

- De tipo epigráfico sobre madera, mármol o yeso (8).

- De tipo arquitectónico incluyendo el análisis tipológico de los yesos ornamentales (9).

- Memorias de restauración donde se refieren cuestiones tipológicas o de cronologías de las mismas (4), para dirimir cuestiones de antigüedad y contemporaneidad.

Además, aunque sin relación con las yeserías del Alcázar, se documentan trabajos más completos y generales sobre la evolución histórica de la construcción con yeso (10) o estudios en torno al yeso y sus aplicaciones (11).

Por último, aunque escasos, en los últimos tiempos son varios los que investigan sobre los problemas del material, existiendo algunos estudios de carácter científico-técnico, ya sean de caracterización de materiales o de su conservación preventiva, entre los que podemos destacar la investigación de Ramón Rubio conservador de la Alhambra (12), diversos estudios llevados a cabo por Navarro Palazón (13) o García Bueno (14), varios trabajos relacionados con las propiedades del yeso (15) (16), manuales del yeso (17) (18) y otros efectuados por los propios autores de este artículo (19).

\section{METODOLOGÍA}

La metodología seguida para el estudio de las yeserías puede dividirse en las siguientes fases:

1. Investigación historiográfica. Basada en el estudio de las fuentes documentales históricas, técnicas, gráficas, científicas y literarias, comentadas en la introducción, y que básicamente corresponde a los textos existentes relacionados con las yeserías del Monumento. Así como también, a las escasas referencias al Patio del Sol halladas en algunos de ellos, a las descripciones que Pavón Maldonado realiza de alguna de sus yeserías y a los estudios técnicos e investigaciones actualizadas correspondientes al material de yeso.

Por otro lado, aunque se carece de una planimetría actualizada completa de este Patio, se ha podido contar con un plano de planta del conjunto atribuido a Vermondo Resta, de 1608 (Archivo General de Simancas), y otro a Van der Borcht, de 1759 (Archivo del Palacio Real de Madrid), donde se puede observar la evolución de este Patio durante el período comprendido entre estas dos fechas.

2. Toma de muestras. Previamente a esta se observó la tipología arquitectónica y constructiva de las yeserías, así como la existencia de actuaciones anteriores discernibles, detectándose tres texturas de terminación con tonalidades diferentes, cada una de éstas con un acabado y una extensión distinta. Por ello se han tomado tres muestras, una por zona, para su caracterización material, realizando previamente una selección de las mismas, buscando la máxima representatividad y el mínimo impacto visual en cada una de ellas. Para sus extracciones, se han utilizado técnicas poco invasivas, obteniéndose una cantidad mínima pero suficiente de muestra para los análisis a realizar.

De las tres áreas determinadas, se ha tomado una muestra YPS2 de la enjuta descrita por Pavón Maldonado, con una coloración algo más oscura que el resto del paño de yeserías, debido a la acción de una humedad accidental localizada, producida hacía ya unos años, que la dota de una coloración distinta a las otras. Otra YPS se ha obtenido de una de las zonas en las que se observó la existencia de una pátina de agua-barro, técnica consistente en la mezcla de agua con arcillas en suspensión, que aplicada sobre la superficie de las yeserías crea un mimetismo difícil de localizar entre las intervenciones realizadas en los últimos 70 años. Esta técnica se utilizaba para patinar piezas nuevas de sustitución (12), dado que
2. Imagen actual de una de las enjutas descritas por Maldonado tomada por los autores. 
Tabla 1. Relación de las muestras tomadas, su ubicación y posible período de construcción

\begin{tabular}{|c|c|c|c|c|}
\hline $\begin{array}{c}\text { No Orden } \\
\text { muestra }\end{array}$ & $\begin{array}{c}\text { Lugar de } \\
\text { ubicación }\end{array}$ & Designación & $\begin{array}{c}\text { Material y } \\
\text { elemento } \\
\text { arquitectónico }\end{array}$ & $\begin{array}{c}\text { Posible período } \\
\text { de construcción y estilo }\end{array}$ \\
\hline 1 & $\begin{array}{c}\text { Patio del Sol } \\
\text { (Cenefa superior } \\
\text { de la arcada) }\end{array}$ & YPS & Yesería en Friso & $\begin{array}{c}\text { Siglo XIII-XIV (i) } \\
\text { Mudéjar }\end{array}$ \\
\hline 2 & $\begin{array}{c}\text { Patio del Sol } \\
\text { (Enjuta descrita } \\
\text { por Pavón M.) }\end{array}$ & YPS2 & $\begin{array}{c}\text { Yesería en } \\
\text { enjuta de Arco }\end{array}$ & $\begin{array}{c}\text { Siglo XIII-XIV (i) } \\
\text { Mudéjar }\end{array}$ \\
\hline 3 & $\begin{array}{c}\text { Patio del Sol } \\
\text { (Zona distinta } \\
\text { a las anteriores) }\end{array}$ & YPS3 & $\begin{array}{c}\text { Yesería en } \\
\text { Arco y pilastra }\end{array}$ & $\begin{array}{c}\text { Siglo XIII-XIV }(i) \\
\text { Mudéjar }\end{array}$ \\
\hline
\end{tabular}

3. Alzado-Sección del conjunto visto desde el Patio, con los puntos de toma de muestras y el área considerada original.

4. Imagen de la cenefa superior que recorre la arcada, de donde se extrajo la muestra YPS. Alzado-Sección del conjunto visto desde el Patio, con los puntos de toma de muestras y el área considerada original.

el yeso que se intentaba imitar se hallaba sucio, lo que le daba un falso tono ocre, por lo que la pátina de agua de barro trataba de igualar este aspecto. La aplicación de esta técnica se debe a la intervención de restauración de estas yeserías mudéjares Ilevada a cabo por Rafael Manzano. La tercera muestra YPS3, corresponde a una reducida superficie (Figura 3), en la que se observó una textura y coloración algo distinta a las anteriores, además de unas manchas de óxidos puntuales repartidas por su superficie.

El lugar de extracción de cada muestra en los distintos paños y el a priori posible período de construcción de las yeserías quedan reflejados en la Tabla 1 y Figura 3.

Caracterización de las yeserías. El análisis químico elemental de las muestras pulverizadas se ha realizando, mediante un espectrómetro de fluorescencia de Rayos X marca Panalytical (modelo AXIOS), que permite un análisis químico cualitativo y cuantitativo en un amplio rango de concentraciones desde componentes mayoritarios a trazas.

ALZADO-SECCIÓN VISTA DESDE EL PATIO

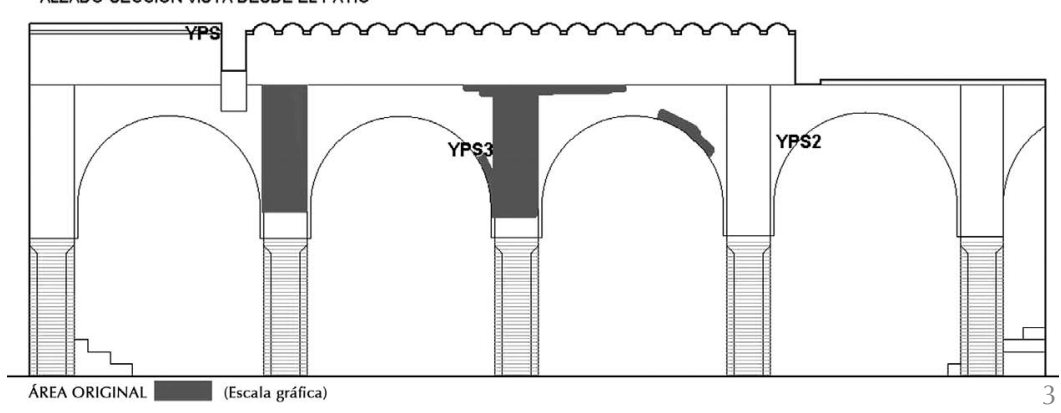

Para cuantificar el contenido de impurezas de los yesos, complementando los resultados de FRX y DRX, se ha determinado el residuo insoluble en agua de las muestras, teniendo en cuenta la ligera solubilidad del yeso dihidrato en agua $\left(2,05 \mathrm{~g} / \mathrm{l}\right.$ a $\left.20^{\circ} \mathrm{C}\right)$, y la de la anhidrita natural $\left(3,0 \mathrm{~g} / \mathrm{l}\right.$ a $\left.20^{\circ} \mathrm{C}\right)(17)$, como fases mayoritarias de los yesos.

El análisis mineralógico de las muestras se ha realizado mediante difracción de rayos $\mathrm{X}$, método de polvo, empleando un difractómetro marca Bruker-AXS modelo D8 Advance, que permite el análisis de las distintas fases cristalinas presentes en las muestras.
Por último, se ha realizado una datación por carbono-14 de la muestra de yeserías YPS (Figura 4), la única que contenía abundantes fibras orgánicas vegetales (estopa, cáñamo o lino) en su masa, probablemente utilizadas para dotarla de unas mejores prestaciones mecánicas.

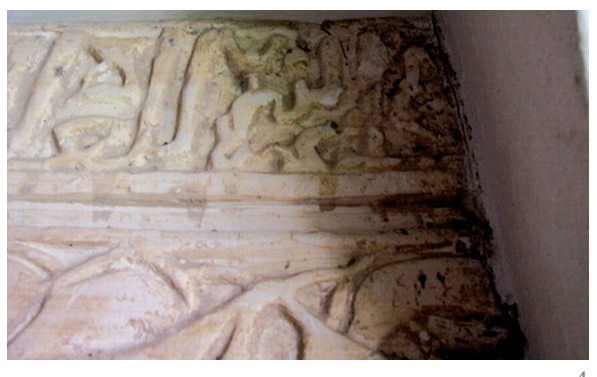

La datación por radiocarbono puede aplicarse de forma genérica a cualquier material de origen orgánico (madera, textiles, papel, restos óseos, etc.), ya que se basa en la desintegración del C-14 presente en los seres vivos, que es incorporado de forma constante mientras viven, y que deja de asimilarse una vez mueren. Existen referencias bibliográficas (20) (21) en las que se utilizan fibras vegetales para datar objetos.

Para eliminar el yeso de la muestra se aplicó un tratamiento con $\mathrm{HCl}$ 0,5 $\mathrm{M}$ que disuelve este sulfato y los carbonatos. Posteriormente se oxida el carbono de las fibras a $\mathrm{CO}_{2}$ y se reduce a carbono elemental con hidrógeno.

La determinación del cociente isotópico 14C/12C que se utiliza para el cálculo de la edad, se ha efectuado combinando la técnica de espectrometría de masas, con un acelerador de partículas Tandetrón AMS (Accelerator Mass Spectrometry), ubicado en el Centro Nacional de Aceleradores de la Cartuja de Sevilla.

En cuanto al error de esta técnica, típicamente puede estar en el orden de 30 años para muestras modernas (2000-3000 años), 100 años para muestras del orden de los 4000-5000 años, y llegaría fácilmente a los 1000 años de error para muestras al límite de detección. Si la muestra es de fecha postnuclear (posterior a 1945), su contenido de C-14 es muy alto, y la curva de calibración tiene una forma muy extrema, que resulta en unos rangos finales bastante estrechos.

\section{RESULTADOS Y DISCUSIÓN}

De acuerdo con la metodología, los resultados obtenidos se han agrupado en dos bloques:

1. Información historiográfica y gráfica. Se ha podido comparar el plano atribuido 

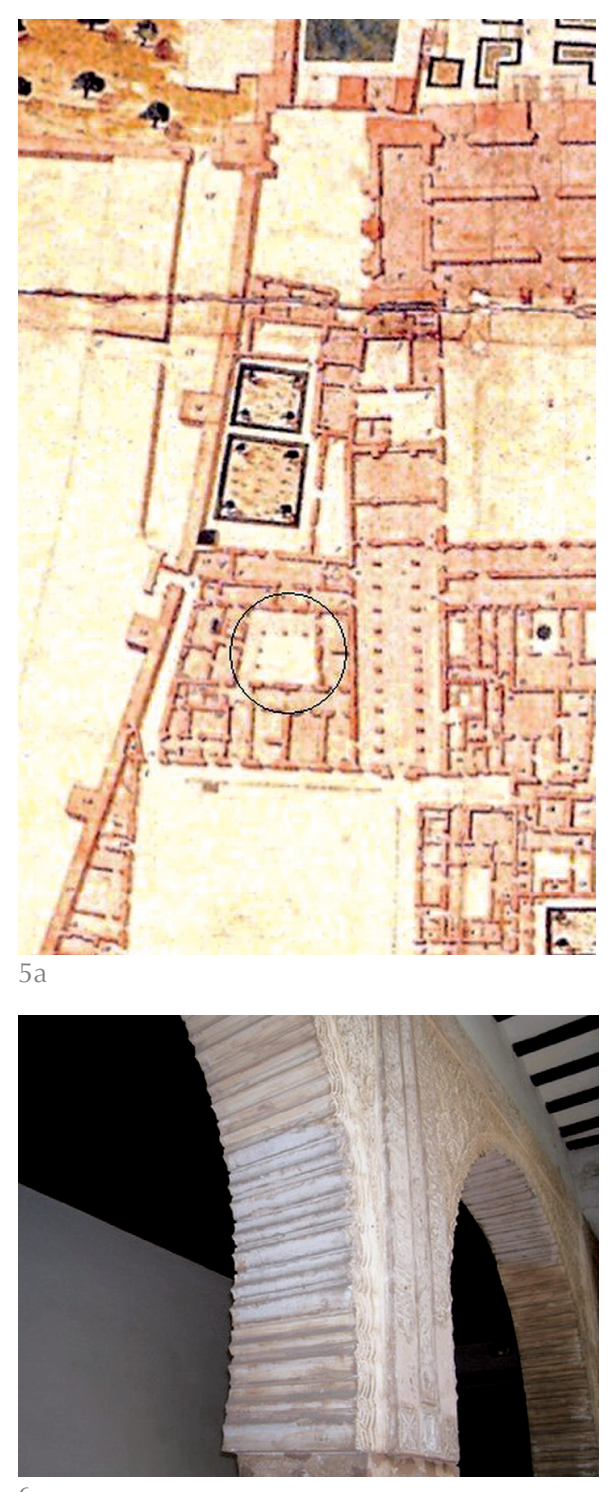

a Vermondo Resta, que nos muestra una planta parcial del Real Alcázar, con otro de V. der Borcht de 1759, apreciándose claramente en el primero la existencia del pórtico mudéjar redescubierto por R. Manzano, así como el ocultamiento de éste en el segundo, y el añadido del pórtico del siglo XVIII recuperado por R. Manzano (Figuras $5 a$ y 5b), que aclaran bastante bien la transformación sufrida en el mismo desde el Mudéjar hasta el Renacimiento.

2. Caracterización de las yeserías. Con respecto al análisis químico de las muestras, los resultados se pueden observar en la Tabla 2, en las que se ha incluido la com-

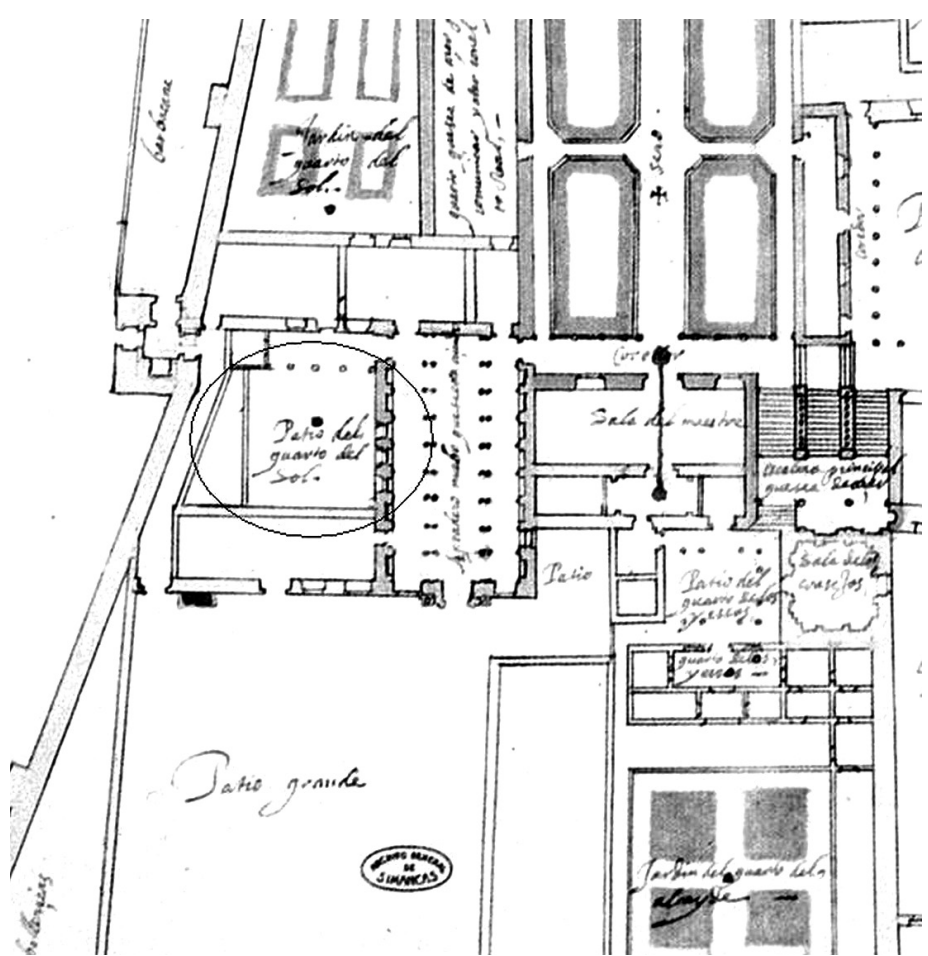

posición de un sulfato de calcio dihidrato químicamente puro (yeso con un índice de pureza del 100\%). Estequiométricamente, si todo el $\mathrm{SO} 3$ analizado fuera atribuible al yeso dihidrato, la riqueza de éste sería de un 98,66\%, 95,05\% y 80,88\% para YPS, YPS2 e YPS3 respectivamente. Se debe tener en cuenta que parte del SO3 analizado puede estar combinado en forma anhidrita, sulfatos de estroncio, magnesio o de alcalinos, por lo que la riqueza final sería inferior.

Se detecta en YPS3 (Figura 6) un incremento en el contenido de Si y en menor medida de $\mathrm{Al}$ y Mg. Estos elementos se podrían asociar a la presencia de cuarzo, magnesita, dolomita, etc. como minerales accesorios del yeso, y que hacen que esta muestra se pueda clasificar como de menor pureza que las otras dos, reforzado también por un contenido en SO3 por debajo del $40 \%$.

Por DRX se ha obtenido la mineralogía de cada una de las muestras, comprobándose una mayor abundancia de fases minerales en la muestra YPS3 (Tabla 3).

En todas las muestras se han identificado trazas de aragonito y dolomita, y en YPS3 además trazas de calcita. El aragonito, es 5a. Planta parcial del Alcázar atribuido a Vermondo Resta (1608)

5b. Planta parcial del Alcázar atribuida a Van der Borcht (1759)

6. Imagen del lugar de extracción de la muestra YPS3.

Tabla 2. Composición química de los elementos mayoritarios y minoritarios de las yeserías (\%)

\begin{tabular}{|c|c|c|c|c|c|c|c|c|c|c|c|c|}
\hline Muestra & $\mathrm{SiO}_{2}$ & $\mathrm{Al}_{2} \mathrm{O}_{3}$ & $\mathrm{Fe}_{2} \mathrm{O}_{3}$ & $\mathrm{MnO}$ & $\mathrm{MgO}$ & $\mathrm{CaO}$ & $\mathrm{Na}_{2} \mathrm{O}$ & $\mathrm{K}_{2} \mathrm{O}$ & $\mathrm{SrO}$ & $\mathrm{SO}_{3}$ & $\mathrm{PC}$ & $\mathrm{TOTAL}$ \\
\hline YPS (1) & 0,34 & 0,11 & 0,07 & 0,00 & 0,32 & 32,43 & 0,04 & 0,04 & 0,20 & 45,89 & 21,82 & 101,05 \\
\hline YPS2 (2) & 0,29 & 0,09 & 0,08 & 0,00 & 0,40 & 31,78 & 0,01 & 0,02 & 0,24 & $\mathbf{4 4 , 2 1}$ & $\mathbf{2 1 , 6 6}$ & 98,54 \\
\hline YPS3 (2) & 3,17 & 0,85 & 0,38 & 0,00 & 1,11 & $\mathbf{3 1 , 3 5}$ & 0,03 & 0,29 & 0,15 & 37,62 & $\mathbf{2 3 , 2 9}$ & 98,07 \\
\hline $\begin{array}{c}\text { Yeso puro de } \\
\text { referencia }\end{array}$ & - & - & - & - & - & 32,57 & - & - & - & $\mathbf{4 6 , 5 0}$ & $\mathbf{2 0 , 9 3}$ & 100 \\
\hline
\end{tabular}

++++ Muy abundante +++ Abundante ++ Medio + Indicios - No detectado 
Tabla 3. Composición mineralógica de las yeserías

\begin{tabular}{|c|c|c|c|c|c|c|}
\hline Mineral & $\begin{array}{c}\text { Yeso } \\
\text { (Sulfato de calcio dihidrato) } \\
\mathrm{CaSO}_{4} \cdot 2 \mathrm{H}_{2} \mathrm{O}\end{array}$ & $\begin{array}{c}\text { Anhidrita } \\
\mathrm{CaSO}_{4}\end{array}$ & $\begin{array}{c}\text { Aragonito } \\
\mathrm{CaCO}_{3}\end{array}$ & $\begin{array}{c}\text { Calcita } \\
\mathrm{CaCO}_{3}\end{array}$ & $\begin{array}{c}\text { Dolomita } \\
\mathrm{CaMg}_{\left(\mathrm{CO}_{3}\right)_{2}}\end{array}$ & $\begin{array}{c}\text { Cuarzo } \\
\mathrm{SiO}_{2}\end{array}$ \\
\hline YPS & ++++ & - & + & + & $+\begin{array}{c}\text { Celestina } \\
\mathrm{SrSO}_{4}\end{array}$ \\
\hline YPS2 & ++++ & + & + & + & + \\
\hline YPS3 & ++++ & + & + & ++ & + \\
+
\end{tabular}

una variedad polimórfica del carbonato de calcio menos estable que la calcita, que se presenta accidentalmente en terrenos sedimentarios, normalmente asociados al yeso o a la celestina en margas o arcillas, por lo que muy probablemente, se trata de una impureza procedente de la piedra de yeso o aljez, al igual que la magnesita. La calcita detectada, es un carbonato que podrían tener un triple origen, por un lado puede tratarse de impurezas presentes en la materia prima (al igual que el aragonito y la dolomita) (18), por otro deberse a materias incorporadas al proceso de elaboración de la pasta (como marmolina), y por último, pueden proceder de la carbonatación de la cal (cálcica o magnésica) que se pudiera haber añadido intencionadamente al yeso. Teniendo en cuenta que se encuentran como trazas o indicios, sería más lógico pensar en que su presencia fuera debida a su incorporación como impureza del aljez.

Por último, la celestina o celestita, es un sulfato de estroncio anhidro con propiedades similares a la barita, ambos minerales con una alta presencia en la península. Existen dos tipos de mineralizaciones de celestina, interesándonos una primaria, que se presenta en niveles de rocas carbonatadas, con nódulos de 2 a $3 \mathrm{~mm}$, constituidos por yeso o por yeso o celestina. En este caso, la transformación aragonito a calcita/dolomita liberaría cantidades relativamente importante de estroncio que al reaccionar con los nódulos de yeso originarían celestina (22).

Del análisis químico y mineralógico de las muestras se deduce, que las yeserías son pastas de yeso, formadas fundamentalmente por sulfato cálcico rehidratado, siendo la muestra YPS3 la que tiene mayor cantidad y diversidad de impurezas.

Tabla 4. Datación mediante C14 realizada por el Centro Nacional de Aceleradores

\begin{tabular}{|c|c|c|c|c|}
\hline \multicolumn{2}{|c|}{ Código Muestra: } & & Código CNA: & CNA300 \\
\hline \multicolumn{2}{|c|}{ Tratamiento aplicado: } & \multicolumn{3}{|c|}{ Limpieza AAA } \\
\hline \multicolumn{3}{|c|}{ Edad Radiocarbono Convencional: } & \multicolumn{2}{|l|}{ NA } \\
\hline$\delta^{13} \mathrm{C}:$ & $-9.4 \pm 1.1 \%$ & pM: & $152.4 \pm 1.2(\mathrm{MC}$ & \\
\hline \multicolumn{3}{|c|}{$\begin{array}{l}\text { Calibración } 1 \sigma(68 \% \text { probabilidad): } \\
\text { [Comienzo: Fin] Área relativa }\end{array}$} & \multicolumn{2}{|c|}{$\begin{array}{l}{[1964 \text { (May) - 1964(Jun)]0.053255 }} \\
{[1964 \text { (Sep) - 1964(Oct)]0.058772 }} \\
{[1968 \text { (Aug) - 1968(Oct)]0.064119 }} \\
{[1969 \text { (Apr) - 1970(Sep)]0.792154 }} \\
{[1971 \text { (Feb) - 1971(Mar)]0.031700 }}\end{array}$} \\
\hline \multicolumn{3}{|c|}{$\begin{array}{l}\text { Calibración } 2 \sigma(95 \% \text { probabilidad): } \\
\text { [Comienzo: Fin] Área relativa }\end{array}$} & \multicolumn{2}{|c|}{$\begin{array}{l}{[1964(\text { Apr) }-1964(\text { Oct) }] 0.112595} \\
{[1968(J u l)-1971 \text { (Jun)]0.887405 }}\end{array}$} \\
\hline
\end{tabular}

Para cuantificar el contenido en impurezas del yeso, se ha aprovechado la ligera solubilidad en agua a $20^{\circ} \mathrm{C}$ del yeso dihidrato y anhidrita, y la insolubilidad de las fases identificadas como impurezas (cuarzo, aragonito, dolomita, calcita, magnesita, celestina). Concretamente, la relación en orden decreciente de las impurezas insolubles en agua obtenidas han sido: 3,80\% para YPS, $3,73 \%$ para YPS2 y $17,29 \%$ en YPS3.

El origen de las impurezas en el yeso puede ser triple: las contenidas en la propia materia prima (aljez), las incorporadas durante las etapas de cocción y posterior trituración en el suelo, y las procedentes del proceso de amasado si se ha realizado también en él, siendo probablemente el primero el más lógico.

El mayor contenido de impurezas podría ser indicativo de una mayor antigüedad al relacionarlo con una elección de la materia prima, fabricación y/o preparación artesanal de las pastas menos cuidadas, dado que estos procesos han ido mejorando con el tiempo (al menos hasta la Revolución Industrial). Este hecho se ha comprobado en la tesis doctoral de uno de los autores de este artículo (23), al analizarse 19 muestras de yeserías correspondientes a los períodos almohade y mudéjar, observándose que en la mayoría de los casos, las más antiguas tienen un mayor contenido de impurezas, no descartándose que este hecho pudiera deberse a una inadecuada selección de la materia prima. Esto indica que además de la influencia de la falta de homogeneidad del aljez de las canteras, pudiera haber una inadecuada selección de la materia prima e incluso la contaminación con impurezas en el proceso de triturado del yeso cocido en el suelo por parte de los yeseros almohades y mudéjares.

Con respecto a la datación mediante C14 de las fibras vegetales halladas en YPS, se muestra a continuación el resultado obtenido en el CNA (Tabla 4).

Se puede observar, en primer lugar, como los rangos de datación son muy estrechos, al tratarse de una muestra del período postnuclear con un contenido en C-14 muy elevado. La cronología más probable de estas fibras oscila entre junio de 1968 y junio de 1971, por lo que se puede afirmar que al 
menos esta zona de la yesería del Patio se restauró en esa fecha (Figura 4), correspondiendo con las mismas en las que R. Manzano restauró la sebka del Patio del yeso, entre los años 1969 y 1971.

Referente a la muestra YPS2, tomada de la enjuta descrita por Pavón Maldonado, nos inclinamos a pensar que se trata de una restauración, posiblemente realizada igualmente por R. Manzano en la misma época que la anterior YPS, a la vista del ensayo organoléptico realizado al paramento, reforzado por los análisis químicos, mineralógicos y datos de impurezas insolubles obtenidos. Este extremo quedó confirmado cuando, se limpió la zona de toma de muestra y se observó que esta yesería tiene unos $2 \mathrm{~cm}$. de espesor y apoya sobre una base de ladrillo cerámico con restos de mortero de cemento (Figura 7).

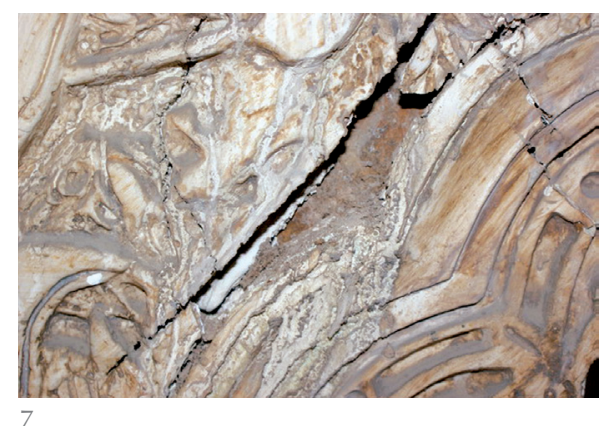

Con respecto a la muestra YPS3, sus resultados de caracterización invitan a considerar que sí se trate de una zona original, según se ha comentado, constatándose además la presencia en diversas zonas de clavos forjados (Figura 8), según técnica de aplicación descrito por Rubio Domene (24) para la colocación de placas moldeadas en época nazarí (1238-1492), sistema utilizado también durante el mudéjar sevillano.

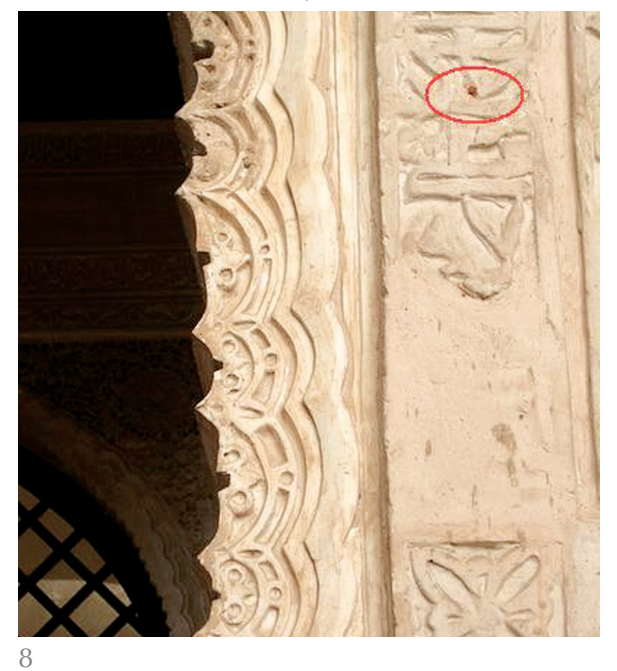

Durante este período, las técnicas más usadas fueron la talla o cuchillo y el molde. En la primera, sobre el yeso fresco alisado con el fratás, se trazaba una retícula cuadriculada, que luego se vaciaba, retallaba y coloreaba, adoptando las diversas formas del ataurique. En la segunda, el uso de moldes permitía una labor más rápida, aunque una última faena se hacía también a cuchillo, permitiendo mostrar así la huella personal del artesano en los detalles (25).

\section{CONCLUSIONES}

A pesar de la escasa información existente de este patio y la falta de una clara diferenciación en la restauración de las yeserías de este pórtico tras la búsqueda del mimetismo entre las yeserías originales y restauradas una vez aplicada la técnica de agua-barro, mediante la metodología historiográfica y de caracterización aplicada, ha sido posible plantear una secuencia de la reconstrucción de estas yeserías al identificar, datar y acotar las zonas originales y las restauradas de estas yeserías mudéjares del siglo XIII.

Del análisis químico y mineralógico se deduce que todas las yeserías son pastas de yeso, formadas fundamentalmente por sulfato cálcico rehidratado (dihidrato) con más o menos impurezas, sin adición intencionada de cal o arena, descartándose que pudiera tratarse de morteros de yeso o morteros bastardos de yeso y cal, observándose igualmente una mayor abundancia de minerales distintos en el yeso de la muestra YPS3. Con respecto al residuo insoluble, las yeserías con un contenido inferior (YPS e YPS2) podrían corresponder a realizaciones más modernas de restauración, por lo que no sería de extrañar una selección de las materias primas y/o elaboración de los yesos cocidos más depurada, mientras que porcentajes bastante superiores (YPS3), pueden ser indicativos de lo contrario, acercándonos a una época más antigua y artesanal.

Por último la datación con Carbono 14 realizada a las fibras halladas en YPS, sitúan su ejecución con una más alta probabilidad entre 1968 y 1971 . Esta metodología junto a las técnicas descritas, ampliadas con otras si ello fuera necesario, según los casos, podría aplicarse a yeserías de otros edificios o distintos períodos históricos, para obtener la máxima información de ellas.
7. Imagen de la base tras la limpieza de la zona de extracción de la muestra YPS2.

8. Imagen de las manchas de óxido debido a los clavos ocultos junto a la muestra YPS3. 


\section{REFERENCIAS}

(1) Hernández Núñez, J.C., Morales, A.J. (1999). El Real Alcázar de Sevilla. Scala Publishers Ltd. (1. a ed.). Imp. en España por Fournier A. Gráficas, S.A., London.

(2) Lleó Cañal, V. (2002). El Real Alcázar de Sevilla. Patronato del Real Alcázar: Lunwerg, D.L.

(3) Manzano Martos, R. (2003). Los Conservadores Municipales del Real Alcázar. p. 111, Patronato del Real Alcázar de Sevilla.

(4) Baceiredo Rodríguez, I., López Madroñero, M.J. (2003). Restauración del hueco almohade del patio del Yeso, Apuntes del Alcázar de Sevilla,4. Sevilla.

(5) Pavón Maldonado, B. El Arte Hispanomusulmán en su Decoración Floral, Ma: Mateu Cromo, S.A. Pinto, 2. ${ }^{a}$ edición aumentada, M.A.E., Agencia Española de cooperación Internacional y Dirección General de Bellas Artes y Archivos del Ministerio de Cultura. (1. a Edición, Madrid, 1981) 1990, Tablas VIII-XIII-23, Fig. 207 y Tabla XX-51, Fig. 388.

(6) Valle Fernández, T. y Respaldiza Lama, P. J. (2000). La pintura Mural Almohade en el Palacio del Yeso. Apuntes del Alcázar de Sevilla,1. Patronato del Real Alcázar de SeviIla. Sevilla.

(7) Tabales Rodriguez, M. Á. (2003). Investigaciones Arqueológicas en el Alcázar de SeviIla. Campaña 2000. Anuario Arqueológico de Andalucía 2000, Consejería de Cultura de la Junta de Andalucía, pp. 45-69. ISBN: 84-8266-330-5, Sevilla.

(8) Cano Ávila, P., Aly Tawfik M. E. (2004). Estudio Epigráfico-Histórico de las Inscripciones árabes de los portalones y ventanas del Patio de las Doncellas del Palacio de Pedro I, Apuntes del Alcázar de Sevilla, 5. Patronato del Real Alcázar de Sevilla, Sevilla.

(9) Pavón Maldonado, B. (2004). Tratado de Arquitectura Hispanomusulmana, Madrid. Consejo Superior de Investigaciones Científicas.

(10) Villanueva Domínguez, L. (2004). Evolución histórica de la construcción con yeso. Informes de la Construcción, 56(493): 5-12.

(11) Díez Reyes, M. C. (2004). En torno al yeso. Informes de la Construcción,56(493):13-18.

(12) Rubio Domene, R. (2010). Yeserías de la Alambra. Historia, Técnica y Conservación, Universidad de Granada-Patronato de la Alambra y el Generalife.

(13) Navarro Palazón, J., Jiménez Castillo, P. (2005). La Yesería en época Almohade. Estudios Árabes e Islámicos: Monografías, 11. Patrice Cressier, Maribel Fierro y Luís Molina editores, CSIC, Madrid.

(14) García Bueno, A., Medina Florez, V. J., González Segura, A. (2006). La policromía de los fragmentos de yeso almacenados en los depósitos del Museo de la Alhambra. 16th International Meeting on Heritage Conservation.

(15) P. Coquard, R. Boistelle. (1994). Hardness, elasticity modulus and flexion strength of dry set plaster. Journal of Materials Science, 29: 4611-4617.

(16) Q.L. Yu, H.J.H. Brouwers. (2001). Microstructure and mechanical properties of b-hemihydrate produced gypsum: An insight from its hydration process. Construction and Building Materials, 25: 3149-3157.

(17) Villanueva Domínguez, L., García Santos, A. (2001). Manual del Yeso, Madrid. Asociación Técnica y Empresarial del Yeso ATEDY-DOSSAT 2000.

(18) Wirsching, F. (1996). Sulfato de Calcio. Resumen de la Ullmann Enciclopedia of Industrial Chemistry, Vol. A 4 (5. ${ }^{a}$ Edición), VCH Verlagsgesellschaft mbH D-69451 Weinheim, Germany, Traducido y editado por Atedy.

(19) Blasco López, F.J., Alejandre Sánchez, F.J. (2010). Caracterización de yeserías de tradición islámica del Real Alcázar, Sevilla. ISBN: 978-84-614-2982-0, Océ, España.

(20) Thomas J. Conolly, William J. Cannon. (1999). Comments on America’́s oldest Basketry. Radiocarbon,41(3): 309-313.

(21) Shozo Mihara, Kazuo Miyamoto, Hidefumi Ogawa, Teiji Kurosaka, Toshio Nakamura, Hiroko Koike. (2004). Ams 14C Dating using black Pottery and Fiber Pottery. Radiocarbon, 46(1): 407-412.

(22) C.S. De Galdeano, M. Ortega, J. Rodríguez y N. Velilla. (1984). Mineralizaciones de celestina en olistostromas de la depresión del Guadalquivir. Boletín Geológico y Minero, 95(3): 268-275.

(23) Blasco López,F.J. (2011). Yeserías medievales de tradición islámica del Real Alcázar de Sevilla: Revisión historiográfica, metodología para la caracterización, evaluación de su durabilidad y elaboración de un inventario, Universidad de Sevilla.

(24) Rubio Domene, R. (2002) Fijación de paños y yeserías en el periodo nazarí de la Alhambra de Granada, Granada. Rubio Domene, R. F. [ed.], ISBN: 84-932568-1-1.

(25) Cómez, R. (1996) El Alcázar del Rey Don Pedro, Sevilla. Diputación Provincial de Sevilla. 